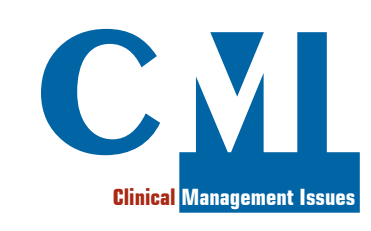

\title{
Polymyositis, Dermatomyositis, and Statins: A Review
}

\begin{abstract}
BACKGROUND: Statins are a well-recognized cause of a variety of skeletal myopathic effects, which generally resolve when discontinuing the treatment. Among autoimmune manifestations associated with statins, there are typical polymyositis (PM) and typical dermatomyositis (DM). OBJECTIVE: To perform a review on published case reports and case series about statin-associated PM and DM.

METHODS: This literature comprehensive search was conducted mainly on PubMed, but also congress abstracts and university websites were considered. Given the paucity of cases, the search was extended to include articles in all languages with English abstract.

RESULTS: Twenty-eight PM and 30 DM cases have been described with prevalence in female (64\%) and senile age. The drugs most frequently involved were atorvastatin and simvastatin. The differential diagnosis should be made among the main myositis subtypes:immuno-mediated necrotizing myopathy (IMNM), inclusion body myositis (IBM), and overlap syndrome with myositis (OM), including anti-synthetase syndrome (ASS).

CONCLUSIONS: Even though the onset of polymyositis or dermatomyositis is a rare phenomenon, it is advisable to consider their presence in patients taking statins and with a non-reversible elevation of creatine phosphokinase.
\end{abstract}

Keywords: Statins; Dermatomyositis; Polymyositis; Autoimmune Myositis

Polimiosite, dermatomiosite e statine: una revierw

CMI 2018; 12(1): 89-102

https://doi.org/10.7175/cmi.v12i1.1364

\section{INTRODUCTION}

\section{Statins}

Due to the wide diffusion of statins, which have the undoubted merit of increasing the survival rate in patients affected by cardiovascular disease, particular attention should be paid to their side effects. The most common ones are toxic myopathies, affecting $2-20 \%$ of patients [1], that generally resolve after drug discontinuation. The risk of statin myopathy and creatine phosphokinase $(\mathrm{CPK})$ increase is dose-dependent.

\section{Polymyositis and Dermatomyositis}

Polymyositis (PM) and dermatomyositis (DM) are idiopathic inflammatory myopa- thies (IIMs) that may be related to statins. All forms of IIMs are considered rare diseases. In USA, DM has prevalence of 1-6 patients per 100,000 persons, while $\mathrm{PM}$ has a prevalence $\sim 10$ per 100,000 [2].

In this context, some cases of association of statins with polymyositis [3-16] and with dermatomyositis [10-12,15,17-35] have been described.

A rate of exposure to statins up to $48 \%$ was found among patients with PM or DM over 50 years of age [10].

The outdated, but still used, diagnostic criteria for PM and DM, according to Bohan and Peter [36,37], are:

- Increase in muscle enzymes;

- Weakness of proximal muscles;
Former Department of Internal Medicine, Ospedali Riuniti Padova Sud Monselice - Padova, Italy
Corresponding author Dr. Mauro Turrin Monselice - Padova Tel.: +393289032440 m.turrin@libero.it

Received: 6 June 2018 Accepted: 5 December 2018 Published: 19 December 2018 
Table I. Criteria for the diagnosis of polymyositis and dermatomyositis. Modified from [36,37]

\begin{tabular}{|c|c|c|}
\hline \multicolumn{2}{|c|}{ Polymyositis } & Dermatomyositis \\
\hline Definite & 4 criteria & 3 criteria \\
Probable & 3 criteria & 2 criteria \\
Possible & 2 criteria & 1 criterion \\
\hline
\end{tabular}

\begin{tabular}{|c|c|c|c|c|c|}
\hline & DM, JDM & NM & PM & DM, ASS & IBM \\
\hline $\begin{array}{l}\text { Onset and } \\
\text { disease course }\end{array}$ & $\begin{array}{l}\text { Acute/subacute } \\
\text { onset; short, benign } \\
\text { or severe, chronic } \\
\text { courses }\end{array}$ & $\begin{array}{l}\text { Acute/subacute } \\
\text { onset; chronic, } \\
\text { slow progression } \\
\text { possible }\end{array}$ & $\begin{array}{l}\text { Acute/subacute } \\
\text { onset; variable } \\
\text { course }\end{array}$ & $\begin{array}{l}\text { Acute/subacute } \\
\text { onset; mostly } \\
\text { chronic course }\end{array}$ & $\begin{array}{l}\text { Slowly progressive; always } \\
\text { chronic }\end{array}$ \\
\hline $\begin{array}{l}\text { Weakness, } \\
\text { extramuscular } \\
\text { symptoms }\end{array}$ & $\begin{array}{l}\text { Amyopathic/proximal } \\
\text { tetraparesis } \pm \\
\text { dysphagia; specific } \\
\text { skin- and organ } \\
\text { manifestation; } \\
\text { malignancy in adults }\end{array}$ & $\begin{array}{l}\text { Proximal } \\
\text { tetraparesis; rarely } \\
\text { extramuscular } \\
\text { manifestation: } \\
\text { heart, lung; } \\
\text { malignancy }\end{array}$ & $\begin{array}{l}\text { Proximal } \\
\text { tetraparesis } \pm \\
\text { dysphagia. No } \\
\text { extramuscular } \\
\text { manifestation }\end{array}$ & $\begin{array}{l}\text { Proximal } \\
\text { tetraparesis; ASS: } \\
\text { ILD, mechanic's } \\
\text { hands, arthritis, } \\
\text { Raynaud's } \\
\text { syndrome. Other } \\
\text { OM: scleroderma, } \\
\text { SLE }\end{array}$ & $\begin{array}{l}\text { Long finger flexors, knee } \\
\text { extensors, dysphagia }\end{array}$ \\
\hline CPK level & $\begin{array}{l}\text { Normal or around } 10 \\
-50 \text { fold elevated }\end{array}$ & $\begin{array}{l}\text { Around } 10-50 \text { fold } \\
\text { elevated }\end{array}$ & $\begin{array}{l}\text { Around } 10-50 \\
\text { fold elevated }\end{array}$ & $\begin{array}{l}\text { Around } 10-50 \text { fold } \\
\text { elevated }\end{array}$ & Normal to 15 fold elevated \\
\hline Autoantibodies & $\begin{array}{l}\text { Mi-2, MDA5 (ILD), } \\
\text { TIF- } 1 \gamma \text { (malignancy), } \\
\text { NXP2 (malignancy), } \\
\text { SAE }\end{array}$ & $\begin{array}{l}\text { SRP, HMGCR } \\
\text { (malignancy) }\end{array}$ & Unspecific & $\begin{array}{l}\text { ASS: Jo-1, PL-7, } \\
\text { PL-12, HA, EJ, KS, } \\
\text { Zo, OJ } \\
\text { Other OM: Ku, Ro/ } \\
\text { SS-A, SS-B, PM/Scl, } \\
\text { U-snRNP }\end{array}$ & cN1A \\
\hline $\begin{array}{l}\text { Muscle } \\
\text { pathology }\end{array}$ & $\begin{array}{l}\text { Perimysial } \\
\text { inflammation, } \\
\text { perifascicular } \\
\text { atrophy, MHC class } \\
\text { I, complement on } \\
\text { capillaries and/ } \\
\text { or sarcolemma, } \\
\text { capillary loss }\end{array}$ & $\begin{array}{l}\text { Scattered necrosis; } \\
\text { MHC class I, } \\
\text { complement on } \\
\text { capillaries and/or } \\
\text { sarcolemma }\end{array}$ & $\begin{array}{l}\text { Endomysial } \\
\text { CD8+ T cells }\end{array}$ & $\begin{array}{l}\text { Perifascicular } \\
\text { necrosis, MHC class } \\
\text { I and II, complement } \\
\text { on sarcolemma }\end{array}$ & $\begin{array}{l}\text { Endomysial CD8+ T cells, MHC } \\
\text { class I, amyloid, vacuoles, } \\
\text { tubulofilaments, mitochondrial } \\
\text { impairment (COX, paracr, } \\
\text { inclusions) }\end{array}$ \\
\hline $\begin{array}{l}\text { Treatment and } \\
\text { its response }\end{array}$ & $\begin{array}{l}\text { Basic: GS, AZA/MTX/ } \\
\text { MMF; skin and JDM: } \\
\text { IVIG; } \\
\text { Lung/Escal.: RTX, } \\
\text { CYC, IVIG, (CsA); } \\
\text { Mostly good } \\
\text { response except for } \\
\text { malignancy or ILD }\end{array}$ & $\begin{array}{l}\text { Basic: GS, AZA } \\
\text { MTX/MMF; } \\
\text { Lung/Escal.: RTX, } \\
\text { CYC, IVIG; } \\
\text { Overall response } \\
\text { good-moderate, but } \\
\text { escalation often } \\
\text { required }\end{array}$ & $\begin{array}{l}\text { Basic: GS, AZA/ } \\
\text { MTX/MMF; } \\
\text { Escal.: RTX, } \\
\text { CYC, IVIG; } \\
\text { Mostly good } \\
\text { response }\end{array}$ & $\begin{array}{l}\text { Basic: GS, AZA/MTX/ } \\
\text { MMF; } \\
\text { Lung/Escal.: RTX, } \\
\text { CYC, IVIG (CsA); } \\
\text { Mostly good } \\
\text { response except for } \\
\text { malignancy or ILD }\end{array}$ & $\begin{array}{l}\text { No basic immunosuppression; } \\
\text { Probatory IVIG in selected } \\
\text { patients justifiable; } \\
\text { Severe dysphagia: local } \\
\text { botulinum toxin or myotomy, } \\
\text { percutaneous feeding tube. } \\
\text { Usually refractory to } \\
\text { treatment }\end{array}$ \\
\hline
\end{tabular}

Table II. Overview of the clinical presentation, auto-antibodies, muscle pathology and treatment in the main subtypes of myositis. Modified from [41].

$\mathrm{Ab}=$ antibody $; \mathrm{ASS}=$ anti-synthetase syndrome; $\mathrm{AZA}=$ azathioprine $; \mathrm{CD} 8^{+} \mathrm{T}$ cells $=$ cluster of differentiation 8 of cytotoxic $\mathrm{T}$ cells; $\mathrm{cN} 1 \mathrm{~A}=$ anti-cytosilic 5 '-nucleotidase $1 \mathrm{~A} \mathrm{Ab} ; \mathrm{COX}=$ cytochrome oxidase staining in muscle fibers; $\mathrm{CPK}=$ creatine phosphokinase; $\mathrm{CsA}=$ cyclosporine $\mathrm{A} ; \mathrm{CYC}=$ cyclophosphamide; $\mathrm{DM}=$ dermatomyositis; $\mathrm{EJ}=$ anti-glycyl tRNA synthetase $\mathrm{Ab} ; \mathrm{GS}=$ glucocorticoids; $\mathrm{HA}=$ anti-tyrosyl tRNA syntethase $\mathrm{Ab}$;

HMGCR = anti-3-hydroxy-3-methylglutaryl-coenzyme A reductase autoantibodies; IBM = inclusion body myositis; ILD = interstitial lung disease; IVIG = intravenous immunoglobulin G; JDM = juvenile dermatomyositis; Jo-1 = anti-histidil tRNA synthetase $\mathrm{Ab} ; \mathrm{KS}=$ anti-asparaginyl tRNA synthetase $\mathrm{Ab} ; \mathrm{Ku}=$ antibodies against $\mathrm{Ku}$ antigen ( $\mathrm{p} 70$ and $\mathrm{p} 80$ subunits); MDA5 = anti-melanoma differentiation-associated gene $5 \mathrm{Ab} ; \mathrm{MHC}=$ major histocompatibility complex; $\mathrm{Mi}-2$ = anti-chromodomain helicase DNA binding protein $4 \mathrm{Ab} ; \mathrm{MMF}=$ mycophenolate mofetil; MTX = methotrexate; $\mathrm{NM}=$ necrotizing myopathy; $\mathrm{NXP} 2$ = anti-nuclear matrix protein $2 \mathrm{Ab} ; \mathrm{OJ}=$ anti- isoleucyl tRNA synthetase $\mathrm{Ab} ; \mathrm{OM}=$ overlap myositis; PL-7 = anti-threonyl tRNA synthetase Ab; PL-12 = anti-alanyl tRNA synthetase $\mathrm{Ab} ; \mathrm{PM} / \mathrm{Scl}=$ anti-PM-Scl-75 and $\mathrm{PM} / \mathrm{Scl}-100$ polypeptides $\mathrm{Ab} ; \mathrm{Ro} / \mathrm{SS}-\mathrm{A}=$ anti-Sjögren's-syndrome-related antigen A (against the Ro52 and Ro60 autoantigen) Ab; RTX = rituximab; SAE = anti-small ubiquitin-like modifier activating enzyme $\mathrm{Ab} ; \mathrm{SLE}$ = systemic lupus erythematosus; $\mathrm{SRP}=$ anti-signal recognition particle autoantibodies; SS-B = anti-Sjögren's-syndrome-related La antigen Ab; TIF- $\gamma=$ anti-transcription intermediary factor gamma $\mathrm{Ab} ; \mathrm{U}$-snRNP = anti-U1 small nuclear ribonucleoprotein particle $\mathrm{Ab} ; \mathrm{Zo}=$ anti-phenylalanyl synthetase $\mathrm{Ab}$ 
- Electromyographic alterations;

- Bioptic alterations; and

- Characteristic skin rash.

As described in Table I, PM and DM are judged "definite", "probable", or "possible" according to the number of criteria met by the patient.

The classification of inflammatory myopathies has undergone several revisions [38-40] since the earliest descriptions by Bohan and Peter.

Five main subtypes of myositis, i.e. dermatomyositis (DM and juvenile DM), necrotizing myopathy (NM), PM, overlap myositis (OM and anti-synthetase syndrome-ASS), and inclusion body myositis (IBM), have been well described in a recent German review [41] (Table II).

In September 2018, Selva-O'Callaghan and colleagues published on Lancet Neurology a new classification of inflammatory myopathies in the adult [42] based on the clinical characteristics of the main clinical and phenotype-specific autoantibody groups, in which they specified also that the diagnosis of $\mathrm{PM}$ is a diagnosis of exclusion.

In the same period a further classification was published also based on a targeted clinical-serological approach [43]. In reference to the 708 variables (in particular myositis-specific autoantibodies-MSA) collected in 260 adult patients of the French register on myositis, 4 clusters of patients emerged (IBM, IMNM, DM, ASS), while PM did not more found place.

Besides clinical criteria, magnetic resonance imaging (MRI) $[39,44]$ can be use- ful to make the right diagnosis. MRI with whole-body technique (WBMRI) is considered particularly useful for identifying the involvement of muscles: parameters such as inflammation, fibrosis, and atrophy can be used to determine the pattern of disease activity even at subclinical level [45-49]. In addition, MRI guides the choice of the site for muscle biopsy.

Figure 1, coming from a personal case report [50], highlights the typical MRI elements, which are suggestive for DM/PM.

IIMs can be treated with glucocorticoids (initial and basic treatment) and with immunosuppressants (methotrexate, azathioprine, mycophenolate mofetil). An additional or alternative therapy may be undertaken with cyclosporin A (or tacrolimus) or intravenous immunoglobulin, with therapy escalation to rituximab or cyclophosphamide.

Treatment with repository corticotropin injection (RCI) in a recent open label clinical trial [51] was effective, safe and tolerable, and led to a steroid dose reduction in adult patients with myositis refractory to glucocorticoid and traditional immunosuppressive drugs.

In this article, the possible implications of statins in autoimmunity have been thoroughly considered with the support of a literature search.

\section{LITERATURE SEARCH STRATEGY}

In this review, the literature search was conducted mainly on PubMed, but also congress abstracts and universities websites

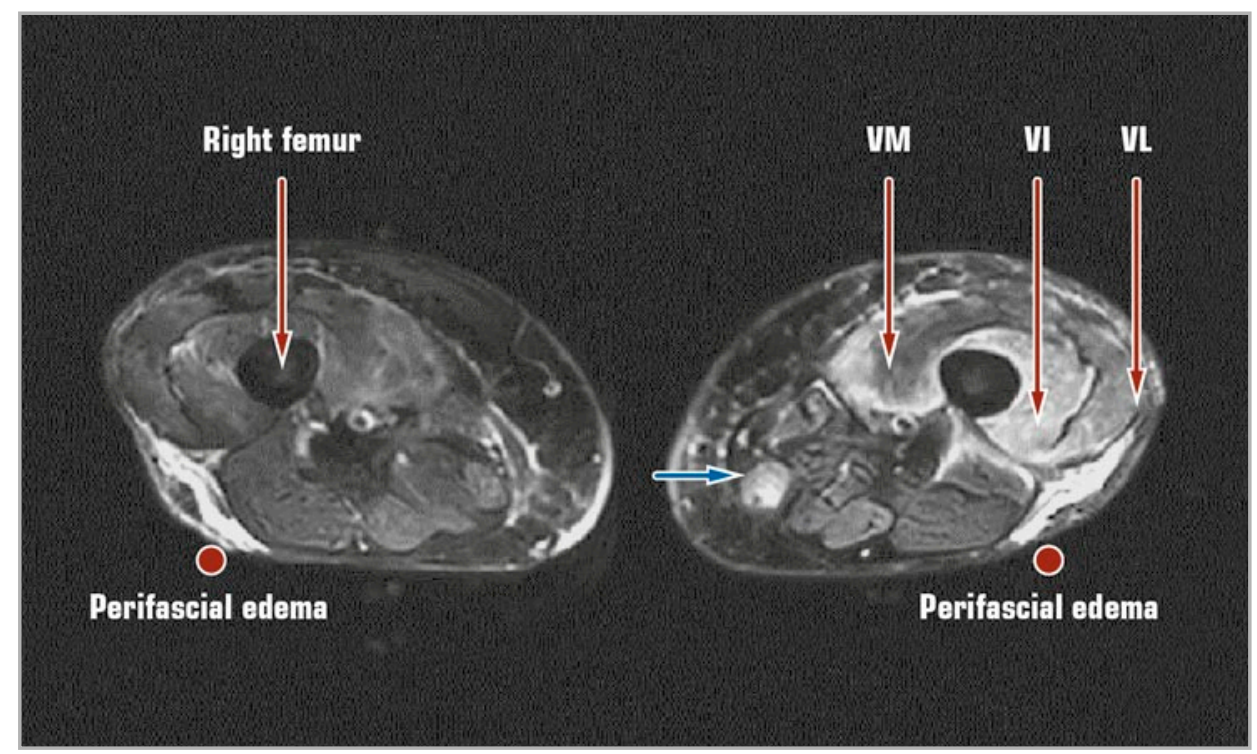

Figure 1. Example of thighs magnetic resonance imaging (MRI). Axial STIR (short-tau inversion recovery sequences) images: axial fat-suppressed MRI images show increased signal intensity. Intense edema of the muscular bundles of both quadriceps, especially on the left, of the large adductor muscle on the left, of the gracilis (blue arrow), and of the semitendinosus muscles. Subcutaneous edema between muscles and fascia lata on both sides. Modified from [50].

$\mathrm{VI}=$ vastus intermedius; $\mathrm{VL}=$ vastus lateralis $\mathrm{VM}=$ vastus medialis 
were considered. The search terms used were: “myositis”, "myopathy”, "statins”, “muscular manifestations”, "dermatomyositis”, "polymyositis", "statin-induced autoimmune myopathy", "creatine (phospho) kinase", "myoglobin", and "troponin". The keywords were combined with the Boolean operators "AND" and "OR". Given the paucity of cases, we extended the search to include articles in all languages with English abstract. The selection of the descriptors was carried out in November 2018. No time limit was set in the search.

\section{RESULTS}

Tables III and IV describe the main characteristics of case reports, related to the exposure to statins in 28 adult patients with polymyositis [3-10,12-14,16] and in 30 adult patients with dermatomyositis [10,12,17-35].

\begin{tabular}{|c|c|c|c|c|c|c|c|c|c|c|c|c|}
\hline 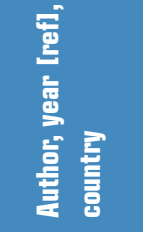 & 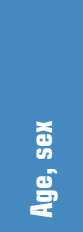 & 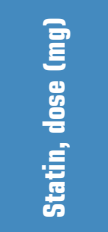 & 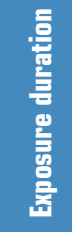 & 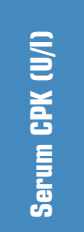 & 둘 홀 홀 & 哼 & 琶 & 总 & 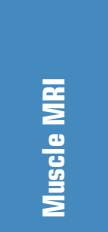 & $\begin{array}{l}\text { 产 } \\
\text { 高 } \\
\text { 昜 }\end{array}$ & 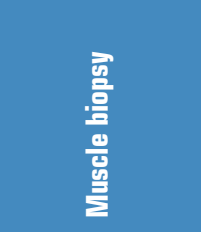 & 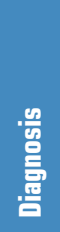 \\
\hline $\begin{array}{l}\text { Giordano, } \\
1997 \text { [6], } \\
\text { Italy }\end{array}$ & 42, M & S 20 & $4 m$ & 503 & n.r. & - & n.p. & $\begin{array}{l}\text { Myogenic } \\
\text { and } \\
\text { neurogenic }\end{array}$ & n.r. & n.r. & $\begin{array}{l}\text { Muscle-fiber } \\
\text { necrosis, } \\
\text { perivascular } \\
\text { and endomysial } \\
\text { inflammation }\end{array}$ & D \\
\hline $\begin{array}{l}\text { Folzenlogen, } \\
\text { २००१ [9], } \\
\text { USA }\end{array}$ & $76, F$ & А २० & n.r. & 9870 & $\begin{array}{l}\text { 1:1280 } \\
\text { speckled }\end{array}$ & $\begin{array}{l}- \\
\text { (anti-Ku } \\
\text { positive) }\end{array}$ & n.p. & Myogenic & n.p. & n.p. & $\begin{array}{l}\text { Muscle cells } \\
\text { necrosis, endo- } \\
\text { mysial mononu- } \\
\text { clear infiltrate, } \\
\text { atrophy fibers }\end{array}$ & D \\
\hline $\begin{array}{l}\text { Riesco- } \\
\text { Eizaguirre, } \\
2003 \text { [7], } \\
\text { Spain }\end{array}$ & $75, M$ & S २० & $6 \mathrm{~m}$ & 6010 & $1: 160$ & n.r. & n.p. & n.r. & n.r. & n.r. & $\begin{array}{l}\text { Muscle fiber } \\
\text { necrosis, } \\
\text { perivascular } \\
\text { inflammation }\end{array}$ & $\mathrm{Pr}$ \\
\hline $\begin{array}{l}\text { Takagi, } \\
2004 \text { [8], } \\
\text { Japan }\end{array}$ & $69, \mathrm{M}$ & P 10 & $2 w$ & 943 & + & + & n.p. & Myopathic & n.r. & n.r & n.r. & $\mathrm{Pr}^{*}$ \\
\hline \multirow[t]{4}{*}{$\begin{array}{l}\text { Fauchais, } \\
2004 \text { [4], } \\
\text { France }\end{array}$} & $56, F$ & S n.r. & $2 \mathrm{~m}$ & 925 & 1:5120 & - & n.p. & Normal & n.r. & n.r. & $\begin{array}{l}\text { Endomysial T } \\
\text { cell infiltration, } \\
\text { type II muscle } \\
\text { atrophy }\end{array}$ & $\mathrm{Pr}$ \\
\hline & $54, F$ & A n.r. & $4 \mathrm{~m}$ & 7400 & - & - & n.p. & Normal & n.r. & n.r. & $\begin{array}{l}\text { T cell infiltration, } \\
\text { type II muscle } \\
\text { atrophy }\end{array}$ & $\mathrm{Pr}$ \\
\hline & $78, F$ & Fn.r. & $4 m$ & 1417 & 1:2150 & $\begin{array}{c}+ \\
\text { (ASS) }\end{array}$ & n.p. & Normal & n.r. & n.r. & $\begin{array}{l}\text { T cell infiltration, } \\
\text { type II muscle } \\
\text { atrophy }\end{array}$ & $\mathrm{Pr}$ \\
\hline & $68, M$ & Fn.r. & $7 \mathrm{~m}$ & 2517 & 1:5120 & + & n.p. & Normal & $\begin{array}{l}\text { Muscle } \\
\text { necrosis }\end{array}$ & n.r. & $\begin{array}{l}\text { Non-contrib- } \\
\text { utive }\end{array}$ & Po \\
\hline \multirow[t]{2}{*}{$\begin{array}{l}\text { Wu Y, } 2014 \\
\text { [5], Canada }\end{array}$} & 57, M & $\begin{array}{c}\text { n.r. } \\
\text { unknown }\end{array}$ & u.k. & 3831 & n.r. & n.r. & n.p. & $\begin{array}{l}\text { Irritable } \\
\text { myopathy }\end{array}$ & n.p. & n.p. & $\begin{array}{l}\text { Mononuclear } \\
\text { inflammatory } \\
\text { infiltration } \\
\text { around intact } \\
\text { muscle fiber }\end{array}$ & $D$ \\
\hline & $56, F$ & A 10 & Few y & 946 & n.r. & n.r. & n.p. & $\begin{array}{l}\text { Irritable } \\
\text { myopathy }\end{array}$ & n.p. & n.p. & $\begin{array}{l}\text { Mononuclear } \\
\text { inflammatory } \\
\text { infiltration } \\
\text { around intact } \\
\text { muscle fiber }\end{array}$ & $D$ \\
\hline
\end{tabular}




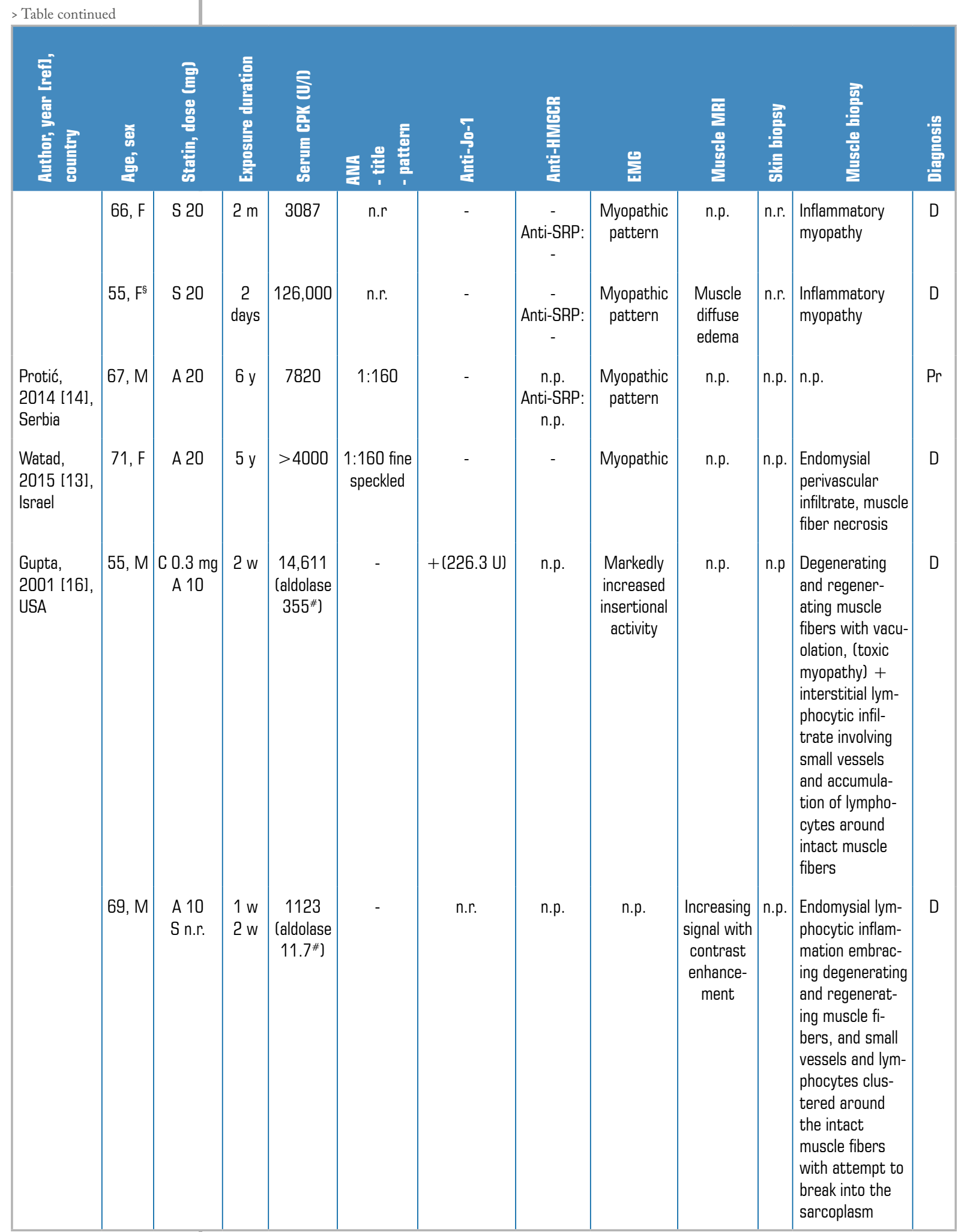

Table III. Statin-associated polymyositis (28 case reports). Most Authors used the diagnostic criteria according to Bohan and Peter [36,37].

* subacute;

$\wedge+$ gemfibrozil

$\S$ ethnicity: African American

\# normal values: $1-7 \mathrm{IU} / 1$

- = negative; + = positive; $\mathrm{A}=$ atorvastatin; $\mathrm{ANA}=$ Anti-nuclear antibody;

anti-HMGCR $=3$-hydroxy-3-methylglutaryl-coenzyme A reductase autoantibodies $;$ anti-Jo-1 = aminoacyl-tRNA

histidyl synthetase autoantibodies; ASS = anti-synthetase syndrome; anti-SRP = anti-signal recognition particle

autoantibodies; $\mathrm{C}=$ cerivastatin; $\mathrm{CPK}=$ creatine phosphokinase; $\mathrm{D}=$ definite; $\mathrm{EMG}$ = electromyography;

$\mathrm{F}=$ fluvastatin HLA-1 = human leukocyte antigen $-1 ; \mathrm{m}=$ months; $\mathrm{MHC}=$ major histocompatibility

complex; $\mathrm{MRI}=$ magnetic resonance imaging; n.p. = not performed; n.r. = not reported $\mathrm{P}=$ pravastatin;

$\mathrm{Po}=$ possible; $\mathrm{Pr}=$ probable; $\mathrm{R}=$ rosuvastatin; $\mathrm{S}=$ simvastatin; $\mathrm{u} . \mathrm{k}$. = unknown; $\mathrm{y}=$ years; $\mathrm{w}=$ weeks 


\begin{tabular}{|c|c|c|c|c|c|c|c|c|c|c|c|c|}
\hline 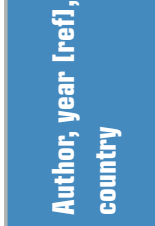 & 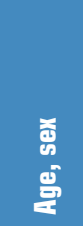 & $\begin{array}{l}\text { 큽 } \\
\text { 兽 } \\
\text { 를 }\end{array}$ & 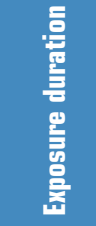 & $\begin{array}{l}\text { 嚍 } \\
\text { 晹尽 }\end{array}$ & 을 善 & 竞 & 盖 & 氮 & 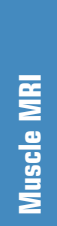 & 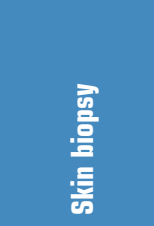 & 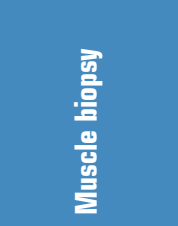 & $\begin{array}{l}\text { 号 } \\
\text { 営 }\end{array}$ \\
\hline $\begin{array}{l}\text { Schalke, } \\
1992 \text { [27], } \\
\text { Germany }\end{array}$ & $66, F$ & P 10 & $5 \mathrm{~m}$ & $>4000$ & $\begin{array}{l}\text { n.r. } \\
\text { n.r. }\end{array}$ & n.r. & n.p. & $\begin{array}{c}\text { Myopathic } \\
\text { changes }\end{array}$ & n.p. & Yes & $\begin{array}{l}\text { T-cell infil- } \\
\text { trates }\end{array}$ & D \\
\hline $\begin{array}{l}\text { Khattak, } \\
1994 \text { [23], } \\
\text { UK }\end{array}$ & 50, M & S n.r. & $6 \mathrm{~m}$ & 1045 & $\begin{array}{c}\text { 1:250 } \\
\text { n.r. }\end{array}$ & n.r. & n.p. & + & n.r. & n.r. & $\begin{array}{l}\text { Lymphocytic } \\
\text { infiltration, } \\
\text { muscle de- } \\
\text { generation }\end{array}$ & $D$ \\
\hline $\begin{array}{l}\text { Hill, } 1995 \\
\text { [25], } \\
\text { Australia }\end{array}$ & $76, F$ & S 10 & $18 \mathrm{~m}$ & 1246 & $\begin{array}{c}\text { 1:2560 } \\
\text { n.r. }\end{array}$ & - & n.p. & n.r. & n.p. & n.r. & $\begin{array}{l}\text { Myositis, at- } \\
\text { rophy, lym- } \\
\text { phocytic infil- } \\
\text { tration }\end{array}$ & $D$ \\
\hline $\begin{array}{l}\text { Rodriguez- } \\
\text { Garcia, } \\
1996 \text { [24], } \\
\text { Spain }\end{array}$ & $63, F$ & L 20 & $24 \mathrm{~m}$ & Increased & $\begin{array}{l}\text { n.r. } \\
\text { n.r. }\end{array}$ & n.r. & n.p. & $\begin{array}{c}\text { Myopathic } \\
\text { changes }\end{array}$ & n.p. & $\begin{array}{l}\text { Inflammatory } \\
\text { infiltration }\end{array}$ & $\begin{array}{l}\text { Inflammatory } \\
\text { infiltrates, } \\
\text { necrosis }\end{array}$ & $D$ \\
\hline $\begin{array}{l}\text { Noël, } 2001 \\
\text { [21], } \\
\text { Switzerland }\end{array}$ & 44, M & A 10 & $12 \mathrm{~m}$ & $>2000$ & $\begin{array}{c}\text { 1:2560 } \\
\text { nucleolar }\end{array}$ & - & n.p. & n.r. & n.r. & $\begin{array}{l}\text { Keratino- } \\
\text { cytes apop- } \\
\text { tosis }\end{array}$ & $\begin{array}{l}\text { Perifascicular } \\
\text { CD4+ T-cells } \\
\text { infiltrates, } \\
\text { severe ne- } \\
\text { crosis }\end{array}$ & $D$ \\
\hline $\begin{array}{l}\text { Vasconcelos, } \\
\text { २००४ [२०], } \\
\text { USA }\end{array}$ & 68, M & $\begin{array}{l}\text { P } 40 \\
\text { S } 20\end{array}$ & $\begin{array}{c}\text { Few } \\
\text { months }\end{array}$ & 2354 & $\begin{array}{l}\text { n.r. } \\
\text { n.r. }\end{array}$ & n.r. & n.p. & $\begin{array}{c}\text { Myopathic } \\
\text { changes }\end{array}$ & n.r & $\begin{array}{l}\text { Chronic peri- } \\
\text { vasculitis }\end{array}$ & $\begin{array}{l}\text { Necrosis, in- } \\
\text { flammatory } \\
\text { infiltrate, } \\
\text { perifascicular } \\
\text { fiber atrophy }\end{array}$ & D \\
\hline $\begin{array}{l}\text { Zuech, } \\
\text { २००५ [22], } \\
\text { France }\end{array}$ & $69, F$ & P n.r. & $24 \mathrm{~m}$ & 6246 & - & n.p. & n.p. & Normal & n.r. & n.r. & Normal & $\mathrm{D}$ \\
\hline $\begin{array}{l}\text { Thual, } 2005 \\
\text { [26], France }\end{array}$ & 76, M & F n.r. & $2 \mathrm{~m}$ & 500 & - & n.p. & n.p. & n.r. & n.r. & n.r. & $\begin{array}{l}\text { Perivascular } \\
\text { inflammation, } \\
\text { muscle de- } \\
\text { generation }\end{array}$ & $D$ \\
\hline \multirow[t]{5}{*}{$\begin{array}{l}\text { Sailler, } \\
\text { २००८ [10], } \\
\text { France }\end{array}$} & $61, F$ & A n.r. & $7 \mathrm{~m}$ & 288 & - & n.r. & n.p. & Myogenic & n.r. & n.r. & $\begin{array}{l}\text { Atrophic fi- } \\
\text { bers endo- } \\
\text { mysial hyali- } \\
\text { nosis } \\
\text { HLA-1 }^{+}\end{array}$ & $\mathrm{D}$ \\
\hline & $72, F$ & A n.r. & $48 \mathrm{~m}$ & 4200 & $\begin{array}{c}\text { 1:2400 } \\
\text { n.r. }\end{array}$ & n.r. & n.p. & Normal & n.r. & n.r. & $\begin{array}{l}\text { Perivascu- } \\
\text { lar lymphoid } \\
\text { infiltrate, } \\
\text { fiber necro- } \\
\text { sis, atrophy, } \\
\text { HLA-1+ }\end{array}$ & $\mathrm{D}$ \\
\hline & $74, F$ & S n.r. & $>36 \mathrm{~m}$ & n.p. & $\begin{array}{c}\text { 1:1280 } \\
\text { n.r. }\end{array}$ & n.r. & n.p. & Myogenic & n.r. & n.r. & $\begin{array}{l}\text { Fibrosis, } \\
\text { perivascular } \\
\text { lymphoid infil- } \\
\text { trate, HLA-1+ }\end{array}$ & $\mathrm{Pr}$ \\
\hline & $74, F$ & P n.r. & $3 \mathrm{~m}$ & 4400 & $\begin{array}{l}\text { n.p. } \\
\text { n.p. }\end{array}$ & n.p. & n.p. & Normal & n.r. & n.r. & $\begin{array}{l}\text { Fiber necrosis } \\
\text { and atrophy, } \\
\text { perivascular } \\
\text { lymphoid infil- } \\
\text { trate, HLA-1 }{ }^{+} \\
\text {fibrosis }\end{array}$ & $D$ \\
\hline & $84, F$ & P n.r. & $32 \mathrm{~m}$ & 712 & $\begin{array}{c}\text { 1:5000 } \\
\text { n.r. }\end{array}$ & n.r. & n.p. & Myogenic & n.r. & n.r. & \begin{tabular}{|l|} 
Lymphocytic \\
infiltrate, fi- \\
ber necrosis \\
and atrophy, \\
HLA-1+
\end{tabular} & D \\
\hline
\end{tabular}

Table continues > 


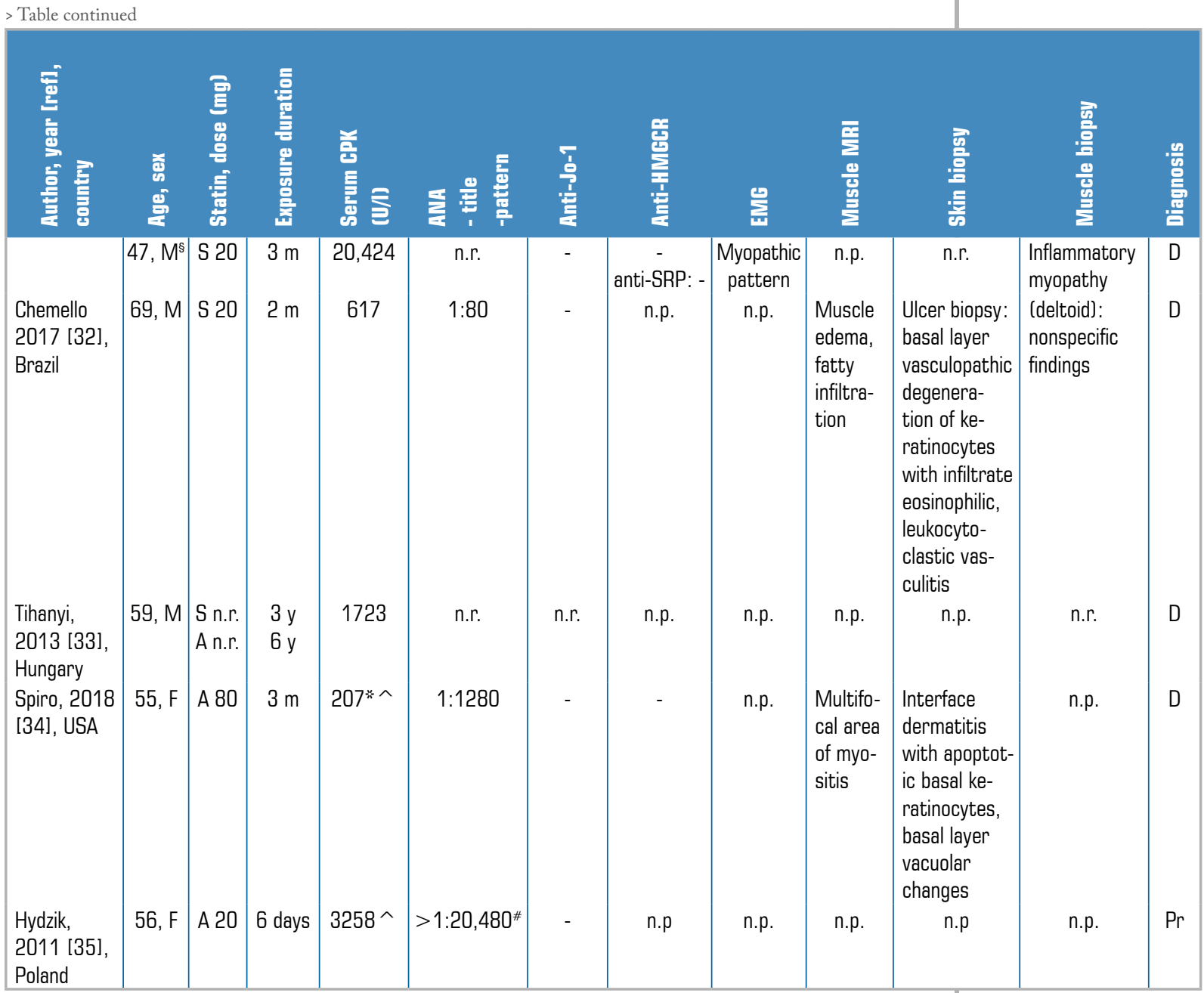

Table IV. Statin-associated dermatomyositis (30 case reports). Most Authors used the diagnostic criteria according to Bohan and Peter [36,37]

$\S$ ethnicity: African American

*aldolase high: peak = $13.8 \mathrm{IU} / 1$

$\wedge$ anti-Mi-2 positive

\#Polish normal values: $<1: 160$

$-=$ negative $;+=$ positive; $\mathrm{A}=$ atorvastatin; $\mathrm{ADM}=$ amyopathic dermatomyositis; $\mathrm{ANA}=$ anti-nuclear

antibody; anti-HMGCR = anti -3-hydroxy-3-methylglutaryl-coenzyme A reductase autoantibodies;

anti-Jo-1 = anti-aminoacyl-tRNA histidyl synthetase autoantibodies; anti-Mi-2 = anti-chromodomain helicase

DNA binding proetin $4 \mathrm{Ab}$; anti-SRP = anti-signal recognition particle autoantibodies; $\mathrm{CPK}=$ creatine

phosphokinase; $\mathrm{D}$ = definite; $\mathrm{EMG}$ = electromyography; $\mathrm{F}$ = fluvastatin; HLA-1 = human leukocyte antigen-1;

$\mathrm{L}=$ lovastatin $; \mathrm{m}=$ months $\mathrm{MRI}=$ magnetic resonance imaging; $\mathrm{n} . \mathrm{p} .=$ not performed; n.r. = not reported;

$\mathrm{P}=$ pravastatin $; \mathrm{Po}=$ possible $; \mathrm{Pr}=$ probable $; \mathrm{R}=$ rosuvastatin $; \mathrm{S}=$ simvastatin; $\mathrm{u} . \mathrm{k}$. = unknown; $\mathrm{y}=$ years

On the basis of the results shown in $\mathrm{Ta}$ bles III and IV, these diseases were more frequent in women $(66 \%)$ and in senile age (the median age at the time of diagnosis was 68 years). The drugs most frequently associated with the disease were atorvastatin and simvastatin in PM and DM, respectively.

The exposure period was very variable: in the PM there was a minimum of two days, in a case concerning an African American patients with CPK peak very high [12], while the maximum exposure time was of 25 years in an elderly woman. In DM, the exposure duration was shorter (median $=6$ months; range $=6$ days -10 years).
The CPK peak was higher in PM (median $=2802 \mathrm{U} / 1 ;$ range $=355-126,000)$ than in DM (median = $1574 \mathrm{U} / \mathrm{l}$; range = 207-20,424), but the difference was not statistically significant.

In one case of dermatomyositis ( 55 years, $\mathrm{F}$, atorvastatin $80 \mathrm{mg}$ ) [34], a normal value of CPK was found associated with increased aldolase. In three further cases, the CPK was lower than $300 \mathrm{U} / 1$.

The determination of ANAs refers only to 33 patients: they were positive in $76 \%$ of patients, i.e. 23 cases (14/18 DM and 9/15 PM).

Anti-HMGCR antibodies were dosed in a total of 9 patients: those described in the 
Brazilian series published in 2018 [12] and the recent case published in October 2018 [34]. All were negative. These antibodies, discovered in 2010 [52,53], are currently being measured in a few specialized centers. Even the anti-signal recognition particle autoantibodies (anti-SRPs), mentioned in the same series, were negative.

In PM, anti-Jo antibodies were negative in 10 out of 14 assays performed. Among the 4 positive patients, one had antisynthetase syndrome. In DM, 12 patients were tested, but none was positive.

We found also a case of polymyositis during therapy with atorvastatin diagnosed after an episode of rhabdomyolysis $(\mathrm{CPK}=14.611 \mathrm{U} / 1$ with myoglobinuria $)$ from cerivastatin, with subsequent detection of positive anti-Jo-1 [16].

\section{DISCUSSION}

According to the literature [11,54-57], statins are involved also in other autoimmune diseases, such as interstitial lung disease, myasthenia gravis, systemic lupus erythematosus, cutaneous lupus, vasculitis, autoimmune hepatitis, lichen planus pemphigoides, and pemphigus erythematosus.

Even more cases appeared in recent years in literature concerning necrotizing myopathies associated with statins and with the coexistence of autoimmune phenomena $[11,55]$.

From 1992 to November 2018 at least 58 defined cases of DM (n. 30) and PM (n. 28), with histological confirmation, associated with exposure to statins were described.

It should be noticed that in the analysis of the South Australian Myositis database including 221 patients with histologically confirmed idiopathic inflammatory myositis, 68 patients ( $30.8 \%$ of cases) were found exposed to statins, at the time of diagnosis: 27/89 PM, 4/23 DM, 12/24 necrotizing myopathy, 20/66 inclusion body myositis, and 5/19 nonspecific chronic inflammatory myositis patients. Exposure to statins was found in $30.3 \%$ of PM and in $17.4 \%$ of DM, thereby highlighting an almost 2-fold increased likelihood compared with controls. However, details on the type of statin, dosage, or duration of statin exposure was not available in this study [58].

Another serious muscle complication associated with statin use has recently been described: autoimmune necrotizing myopathy (IMNM: immune-mediated necrotizing myopathy). It is associated with anti-3-hydroxy-3-methylglutaryl-coenzyme A reductase antibodies (anti-HMGCR) [59].

According to some Authors, most patients affected by necrotizing myopathy with a history of statins, before the discovery of anti-HMGCR were classified as polymyositis. Statin-triggered IMNM and polymyositis would therefore not be two distinct entities, but part of the same pathophysiological spectrum also because they respond well to immunosuppressive treatment $[5,60]$.

Pathognomonic clinical manifestations for dermatomyositis and polymyositis have been reported in three adult patients with autoimmune necrotizing myopathy positive for anti-HMGCR [61-63].

In the IMNM, magnetic resonance detects a characteristic pattern of muscular abnormalities involving mainly hip rotators and glutei: IMNM have significantly more widespread muscle edema, atrophy, and fatty replacement compared with those with polymyositis and dermatomyositis, unlike the fascial edema is more common and widespread in dermatomyositis [44].

In 2013, a Canadian Working Group Consensus defined the main predisposing conditions that promote intolerance or side effects of statins [64]:

- Hypothyroidism;

- Hypovitaminosis D;

- Low body mass index;

- Low CoQ10 enzyme level;

- Excessive use of alcohol;

- Excessive use of cranberry or grapefruit juice;

- Illicit drugs (amphetamine, cocaine, heroin);

- Drug-statin interaction;

- Renal failure;

- Liver failure;

- Diabetes mellitus;

- Biliary obstruction;

- History of pre-existing asymptomatic increase in CPK.

However, a more recent meta-analysis conducted by Nguyen in 2018 found that the significant risk factors for myopathy and/ or rhabdomyolysis associated with statins were [65]:

- Age > 65 years;

- Female gender;

- Diabetes mellitus;

- Renal insufficiency; 
- Cardiovascular disease;

- Drug-drug interactions (clarithromycin, erythromycin, cyclosporin, mibefradil, verapamil, diltiazem, nefazodone, itraconazole, fibrates, gemfibrozil, amiodarone, and protease inhibitors);

- Statin dose;

- Genetic factors (SLCO1B1 gene mutation).

It is useful to dose CPK before starting statin therapy to discover a subclinical myopathy and to exclude, even if very rare, the presence of macro-creatinekinase [66].

Subsequent CPK checks should be done in the third, sixth, and twelfth months because most statin myopathies occur within the first six months of therapy.

Concerning the case with high aldolase [50], it should be pointed out that aldolase dosage is useful because its isolated increase reflects preferential immune-mediated damage affecting early regenerating cells [67].

Regarding the therapy of hypercholesterolemia to be implemented after clinical recovery in patients with IIMs, to my knowledge specific indications do not exist in the literature. Drugs with no side effects on the muscle should be used. In primary prevention, the range of therapy extends from nutraceuticals to ezetimibe. As for nutraceuticals, monacolin $\mathrm{K}$ should not be prescribed because it is chemically identical to lovastatin.

Berberine does not modify CPK [68], while for ezetimibe the incidence of myopathy/rhabdomyolysis was identical (0.2\%) to that found in placebo patients. PCSK9 inhibitors may be used in myopathic patients at high cardiovascular risk. These fully human monoclonal antibodies (e.g. evolucumab, alirocumab), which act against the proprotein convertase subtilisin/kexyn type 9, could be an alternative to statin therapy in severe cases of drug toxicity, such as rhabdomyolysis [1]. Clinical studies comparing statins versus statins + anti-PCSK9 found no differences in muscle and CPK side effects between the two groups $[69,70]$. For patients with idiopathic inflammatory myositis and with coexistent dyslipidemia, anti-cholesterol therapy should be implemented, obviously, with defined drugs without toxic effects on the muscle.

\section{CONCLUSIONS}

The present literature review identified 28 cases of polymyositis and 30 cases of dermatomyositis related to exposure to statins.

To my knowledge, this is currently the widest research about these two rare statin-triggered pathologies.

Keypoints

- Besides the more common side effects on muscle, statins can give rise to autoimmune phenomena

- Inflammatory myopathies are a very heterogeneous group of illnesses that can present with a very different clinical phenotype

- The main subtypes of myositis should be considered in the differential diagnosis: PM, DM, IMNM, OM/ASS, and IBM

- The international literature reports 28 cases of polymyositis and 30 cases of dermatomyositis related to exposure to statins

- It is useful to dose the CPK (and aldolase) before starting statin therapy

- In case of skin rash or muscular symptoms, antinuclear antibody screening is recommended in patients treated with statins

- Patients who have failed to normalize high CPK (> 10 times the upper range of normal) after statin withdrawal and after cortisone therapy should be tested (in addition to ANA and ENA) for myositis-associated and myositis-specific antibodies (MAAs and MSAs), in particular anti-SRP and anti-HMGCR antibodies and, if these are positive, undergo muscle biopsy to confirm the diagnosis of inflammatory or autoimmune myopathy

- Drug therapy is challenging and requires, in addition to corticosteroids, immunosuppressive medications, and intravenous immunoglobulin.

- New anti-PCSK9 monoclonal antibodies may be used in high-risk cardiovascular myopathic patients

- The choice of anti-cholesterol therapy in myopathic patients after clinical recovery is yet not defined

\section{Funding}

This article has been published without the support of sponsors.

Conflicts of interests

The Author declares that he has no conflicts of financial interest regarding the topics covered in this article. 


\section{REFERENCES}

1. Selva-O'Callaghan A, Alvarado-Cardenas M, Pinal-Fernández I, et al. Statin-induced myalgia and myositis: an update on pathogenesis and clinical recommendations. Expert Rev Clin Immunol 2018; 14: 215-24; https://doi.org/10.1080/1744666X.2018.1440206

2. Furst DE, Amato AA, Iorga SR, et al. Epidemiology of adult idiopathic inflammatory myopathies in a U.S. managed care plan. Muscle Nerve 2012; 45: 676-83; https://doi.org/10.1002/ mus.23302

3. Kanth R, Shah MS, Flores RM. Statin-associated polymyositis following omeprazole treatment. Clin Med Res 2013; 11: 91-5; https://doi.org/10.3121/cmr.2012.1110

4. Fauchais AL, Iba Ba J, Maurage $\mathrm{P}$, et al. Polymyositis induced or associated with lipid-lowering drugs: five cases. Rev Med Interne 2004; 25: 294-8; https://doi.org/10.1016/j.revmed.2003.10.013

5. Wu Y, Lach B, Provias JP, et al. Statin-associated autoimmune myopathies: a pathophysiologic spectrum. Can J Neurol Sci 2014; 41: 638-47; https://doi.org/10.1017/cjn.2014.22

6. Giordano N, Senesi M, Mattii G, et al. Polymyositis associated with simvastatin. Lancet 1997; 349: 1600-1; https://doi.org/10.1016/S0140-6736(05)61628-5

7. Riesco-Eizaguirre G, Arpa-Gutierrez FJ, Gutierrez M, et al. Severe polymyositis with simvastatin use. Rev Neurol 2003; 37: 934-6

8. Takagi A, Shiio Y.Pravastatin-associated polymyositis, a case report. Rinsho Shinkeigaku 2004; 44: 25-7

9. Folzenlogen D. A case of atorvastatin combined toxic myopathy and inflammatory myositis. $J$ Clin Rheumatol 2001; 7: 340-5; https://doi.org/10.1097/00124743-200110000-00019

10. Sailler L, Pereira C, Bagheri A, et al. Increased exposure to statins in patients developing chronic muscle diseases: a 2-year retrospective study. Ann Rheum Dis 2008; 67: 614-9; https:// doi.org/10.1136/ard.2007.075523

11. Padala S, Thompson PD. Statins as a possible cause of inflammatory and necrotizing myopathies. Atherosclerosis 2012; 22: 15-21; https://doi.org/10.1016/j.atherosclerosis.2011.11.005

12. Borges IBP, Silva MG, Misse RG, et al. Lipid-lowering agent-triggered dermatomyositis and polymyositis: a case series and literature review. Rheumatol Int 2018; 38: 293-301; https://doi. org/10.1007/s00296-017-3821-3

13. Watad A, Soriano A, Vaknine H, et al. Immune mediated myopathy following long-term statin therapy. Isr Med Assoc J 2015; 17: 128-9

14. Protić D, Baltić S, Stupar NV, et al. A case of myositis with immunological background associated with statin use. Cent Eur J Med 2014; 9: 619-24; https://doi.org/10.2478/s11536-013-0313-z

15. Horák T, Voháňka S, Tvrdíková, et al. Statin-induced necrotizing autoimmune myopathy. Cesk Slov Neurol N2017; 80/113: 569-77; https://doi.org/10.14735/amcsnn2017569

16. Gupta S, Blaivas M, Ike RW, et al. Polymyositis evolving after rhabdomyolysis associated with HMG-CoA reductase inhibitors: a report of two cases. JClin Rheumatol 2001; 7:332-5; https:// doi.org/10.1097/00124743-200110000-00015

17. Zaraa IR, Labbène I, Mrabet D, et al. Simvastatin-induced dermatomyositis in an 50-year-old-man. BMJ Case Rep 2011; 2011: bcr0220113832; http://dx.doi.org/10.1136/ bcr.02.2011.3832

18. Komai I, Takemoto M, Yokote K. Atorvastatin-induced dermatomyositis in an 47-year-old woman with Sjögren's syndrome. Acta Cardiol 2015; 70: 373; https://doi.org/10.1080/ AC.70.3.3080648

19. Rasch A, Schimmer M, Sander CA. Simvastatin-induced dermatomyositis. Hautarzt 2009; 60: 483-9; https://doi.org/10.1007/s00105-008-1637-5

20. Vasconcelos OM, Campbell WW.Dermatomyositis-like syndrome and HMG-CoA reductase inhibitor (statin) intake. Muscle Nerve 2004; 30: 803-7; https://doi.org/10.1002/mus.20127

21. Noël B, Cerottini JP, Panizzon RG. Atorvastatin-induced dermatomyositis. Am J Med 2001; 110: 670-1; https://doi.org/10.1016/S0002-9343(01)00711-2

22. Zuech P, Pauwels C, Dulhoit C, et al. Pravastatin-induced dermatomyositis. Rev Med Interne 2005; 26: 897-902; https://doi.org/10.1016/j.revmed.2005.07.005

23. Khattak FH, Morris IM, Branford WA. Simvastatin-associated dermatomyositis. Br J Rheumatol 1994; 33: 199; https://doi.org/10.1093/rheumatology/33.2.199

24. Rodriguez-Garcia JL, Serrano Commino M. Lovastatin-associated dematomyositis. Postgrad Med J 1996; 72: 694; https://doi.org/10.1136/pgmj.72.853.694

25. Hill C, Zeitz C, Kirkham B. Dermatomyositis with lung involvement in a patient treated with simvastatin. Aust N Z J Med 1995; 25: 745-6; https://doi.org/10.1111/j.1445-5994.1995. tb02870.x

26. Thual N, Penven K, Chevalier JM, et al. Fluvastatin-induced dermatomyositis. Ann Dermatol Venereol 2005; 132: 996-9 
27. Schalke BB, Achmidt B, Toyka K, et al. Pravastatin-associated inflammatory myopathy. NEngl JMed 1992; 327: 649-50; https://doi.org/10.1056/NEJM199208273270919

28. Inhoff $\mathrm{O}$, Peitsch WK, Paredes BE, et al. Simvastatin-induced amyopathic dermatomyositis. Br J Dermatol 2009; 161: 206-8; https://doi.org/10.1111/j.1365-2133.2009.09205.x

29. Cannon CS. Statin-induced rhabdomyolysis and dermatomyositis: a rare combination. JAAPA 2012; 25: 30,32-3; https://doi.org/10.1097/01720610-201208000-00006

30. Fania L, Didona D, Tonanzi T, et al. Simvastatin-associated dermatomyositis. Dermatol Ther 2017; 30. Epub 2017 Mar 14. https://doi.org/10.1111/dth.12480

31. Oztas M, Ugurlu S, Aydin O. Atorvastatin-induced dermatomyositis. Rheumatol Int 2017; 37: 1217-9; https://doi.org/10.1007/s00296-017-3658-9

32. Chemello RML, Benvegnù AM, Dallazem LND, et al. Aggressive and fatal statin-induced dermatomyositis: a case report. OxfMed Case Reports 2017; 12:242-5; https://doi.org/10.1093/ omcr/omx063

33. Tihanyi L, Sutô G, Veress G. Necrotising autoimmune myopathy showing dermatomyositis symptoms during persistent statin treatment. Lege Artis Medicinae 2013; 23: 113-6

34. Spiro J, Butts M.Atorvastatin-induced dermatomyositis: resolution with change in statin? JCR 2018; 24: 406-9; https://doi.org:110.10977/RHU.0000000000000709

35. Hydzik P, Szpak D. Side effects on the HMG-CoA reductase inhibitors (statins). Lupus erythematosus induced by atorvastatin therapy. Przegl Lek 2011; 68: 495-8

36. Bohan A, Peter JB. Polymyositis and dermatomyositis (first of two parts). N Engl J Med 1975; 292: 344-7; https://doi.org/10.1056/NEJM197502132920706; https://doi.org/10.1056/ NEJM197502202920807

37. Bohan A, Peter JB. Polymyositis and dermatomyositis (second of two parts). N Engl J Med 1975; 292: 403-7; https://doi.org/10.1056/NEJM197502132920706; https://doi.org/10.1056/ NEJM197502202920807

38. HoogendijkJE, Amato AA, Lecky BR, et al.119th ENMC international workshop: trial design in adult idiopathic inflammatory myopathies, with the exception of inclusion body myositis, 10-12 October 2003, Narden, The Netherlands. Neuromuscul Disord 2004; 14: 337-45; https:// doi.org/10.1016/j.nmd.2004.02.006

39. Lundberg IE, Tjärnlund A, Bottai M, et al. 2017 European League Against Rheumatism/ American College of Rheumatology classification criteria for adult and juvenile idiopathic inflammatory myopathies and their major subgroups. Ann Rheum Dis 2017; 76: 1955-64; https://doi.org/10.1136/annrheumdis-2017-211468

40. Lilleker JB, Vencovsky J, Wang G, et al. The EuroMyositis registry: an international collaborative tool to facilitate myositis research. Ann Rheum Dis 2018; 77: 30-9; https://doi.org/10.1136/ annrheumdis-2017-211868

41. Schmidt J. Current classification and management of inflammatory myopathies.JNeuromuscul Dis 2018; 5: 109-29; https://doi.org/10.3233/JND-180308

42. Selva-O'Callaghan A, Pinal-Fernández I, Trallero-Araguàs E, et al. Classification and management of adult inflammatory myopathies. Lancet Neurol 2018; 17: 816-28; https://doi. org/10.1016/S1474-4422(18)30254-0

43. Mariampillai K, Granger B, Amelin D, et al. Development of a new classification system for idiopathic inflammatory myopathies based on clinical manifestations and myositis-specific autoantibodies.JAMA Neurol 2018; Sep 10: E1-E10; https://doi.org/10.1001/ jamaneurol.2018.2598

44. Pinal-Fernandez I, Casal-Dominguez M, Carrino JA, et al. Thigh muscle MRI in immune-mediated necrotising myopathy: extensive oedema, early muscle damage and role of anti-SRP autoantibodies as a marker of severity. Ann Rheum Dis 2016; 0: 1-7; http://dx.doi. org/10.1136/annrheumdis-2016-210198

45. Elessawy SS, Abdelsalam EM, Abdel RE, et al.Whole-body MRI for full assessment and characterization of diffuse inflammatory myopathy.Acta Radiol Open 2016; 5:2058460116668216; https://doi.org/10.1177/2058460116668216

46. Huang ZG, Gao BX, Chen H, et al. An efficacy analysis of whole-body magnetic resonance imaging in the diagnosis and follow up of polymyositis and dermatomyositis. PLoSOne 2017; 12: e0181069; https://doi.org/10.1371/journal.pone.0181069

47. Pipitone N. Value of MRI in diagnostics and evaluation of myositis. Curr Opin Rheumatol 2016; 28: 62530; https://doi.org/10.1097/BOR.0000000000000326

48. Day J, Patel S, Limaye V. The role of magnetic resonance imaging techniques in evaluation and management of the idiopathic inflammatory myopathies. Semin Arthritis Rheum 2017; 46: 642-9; https://doi.org/10.1016/j.semarthrit.2016.11.001

49. Tomas X, Milisenda JC, Garcia-Diez AI, et al. Whole-body MRI and pathological findings in adult patients with myopathies. Skeletal Radiol 2018 Oct 30; [Epub ahead of print]; https:// doi.org/10.1007/s00256-018-3107-1 
50. Turrin M, Martinelli S. Statin-induced rhabdomyolysis: polymyositis/dermatomyositis or necrotizing autoimmune myopathy? Description of a clinical case in an elderly patient. G Ital Arterioscler 2017; 8: 83-8

51. Aggarwal R, Marder G, Koontz DC, et al. Efficacy and safety of adrenocorticotropic hormone gel in refractory dermatomyositis and polymoyositis. Ann Rheum Dis 2018; 77: 720-7; https:// doi.org/10.1136/annrheumdis-2017-212047

52. Christopher-Stine L, Casciola-Rosen LA, Hong G, et al. A novel autoantibody recognizing $200-\mathrm{kd}$ and $100-\mathrm{kd}$ proteins is associated with an immune-mediated necrotizing myopathy. Arthritis Rheum 2010; 62: 2757-66; https://doi.org/10.1002/art.27572

53. Mammen AL, Chung T, Christopher-Stine A, et al. Autoantibodies against 3-hydroxy-3-metylglutaryl-coenzyme A reductase in patients with statin-associated autoimmune myopathy. Arthritis Rheum 2011; 63: 713-21; https://doi.org/10.1002/art.30156

54. Noël B. Lupus erythematosus and other autoimmune diseases related to statin therapy: a systematic review. J Eur Acad Dermatol Venereol 2007; 21: 17-24; https://doi.org/10.1111/ j.1468-3083.2006.01838.x

55. Turrin M,Martinelli S. Drug-induced lupus: simvastatin or amiodarone? A case report in elderly. Clinical Management Issues 2013; 7: 17-26; https://doi.org/10.7175/cmi.v7i1.644

56. Lo Schiavo A, Puca RV, Romano F, et al. Pemphigus erythematosus relapse associated with atorvastatin intake. Drug Des Devel Ther 2014; 8: 1463-5; https://doi.org/10.2147/DDDT. S66666

57. Alonso JJ, Jaimez L, Sabio JM, et al. Atorvastatin-induced reversible positive antinuclear antibodies. Am J Med 2002; 112: 329-30; https://doi.org/10.1016/S0002-9343(01)01102-0

58. Caughey GE, Gabb GM, Ronson S, et al. Association of statin exposure with histological confirmed idiopathic inflammatory myositis in an Australian population. JAMA Intern Med 2018; 178: 1224-29; https://doi.org/10.1001/jamainternmed.2018.2859

59. Turrin M. Statins and immune-mediated necrotizing myopathy. Clinical Management Issues 2018; 12: 77-88; https://doi.org/10.7175/cmi.v12i1.1367

60. Mohassel P, Mammen AL. Statin-associated autoimmune myopathy and anti-HMGCR autoantibodies. Muscle Nerve 2013; 48: 477-83; https://doi.org/10.1002/mus.23854

61. Lavian M, Mozaffar T, Goyal N. Clinical dermatomyositis associated with anti-HMC-CoA reductase antibody positive immuno mediated necrotizing myopathy: a case report. Neurology 2017; 88 (16 supplement): P2.125

62. Parikh P,Tavee J, Soltanzadeh P, et al.Anti-3-hydroxy-3-methylglutaryl-coenzyme a reductase autoantibody-positive necrotizing autoimmune myopathy with dermatomyositis-like eruption. Muscle Nerve 2018; 57: E135-E136; https://doi.org/10.1002/mus.26072

63. Dixit A, Abrudescu A. A case of atorvastatin-associated necrotizing autoimmune myopathy, mimicking idiopathic polymyositis. Case Rep Rheumatol 2018; 2018: 5931046; https://doi. org/10.1155/2018/5931046

64. Mancini GB, Tashakkor AY, Baker S, et al. Diagnosis, prevention, and management of statin adverse effects and intolerance: Canadian Working Group Consensus update. Can J Cardiol 2013; 29: 1553-68; https://doi.org/10.1016/j.cjca.2013.09.023

65. Nguyen KA, Li L, Lu D, et al. A comprehensive review and meta-analysis of risk factors for statin-induced myopathy. Eur J Clin Pharmacol 2018; 74: 1099-109; https://doi.org/10.1007/ s00228-018-2482-9

66. Turrin M, Tinello A. Hypercholesterolemia, hyperCKemia and macro-creatine kinase: a case report. Intern Emerg Med 2012: 7 (Suppl 4): 361. S552-S553; https://doi.org/10.1007/ s11739-012-0888-4

67. Casciola-Rosen L, Hall JC, Mammen Al, et al. Isolated elevation of aldolase in the serum of myositis patients: a potential biomarker of damaged early regenerating muscle cells. Clin Exp Rheumatol 2012; 30: 548-53

68. Poli A, Barbagallo CM, Cicero A, et al. Nutraceuticals and functional foods for the control of plasma cholesterol levels. An Intersociety position paper. Pharmacol Res 2018; 134: 51-60; https://doi.org/10.1016/j.phrs.2018.05.015

69. Sabatine MS, Giugliano RP, Keech AC, et al. Evolocumab and clinical outcomes in patients with cardiovascular disease. N Engl J Med 2017; 376: 1713-22; https://doi.org/10.1056/ NEJMoa1615664

70. Szarek M, White HD, Schwartz GG, et al.; ODYSSEY OUTCOMES Committees and Investigators. Alirocumab reduces total nonfatal cardiovascular and fatal events in the ODYSSEY OUTCOMES trial.J Am Coll Cardiol 2018 Oct 27. pii: S0735-1097(18)38961-7. [Epub ahead of print]; https://doi.org/10.1016/j.jacc.2018.10.039 